\title{
To the Centenary of Isaak Markovich Khalatnikov
}

DOI: $10.1134 /$ S1063776119100133

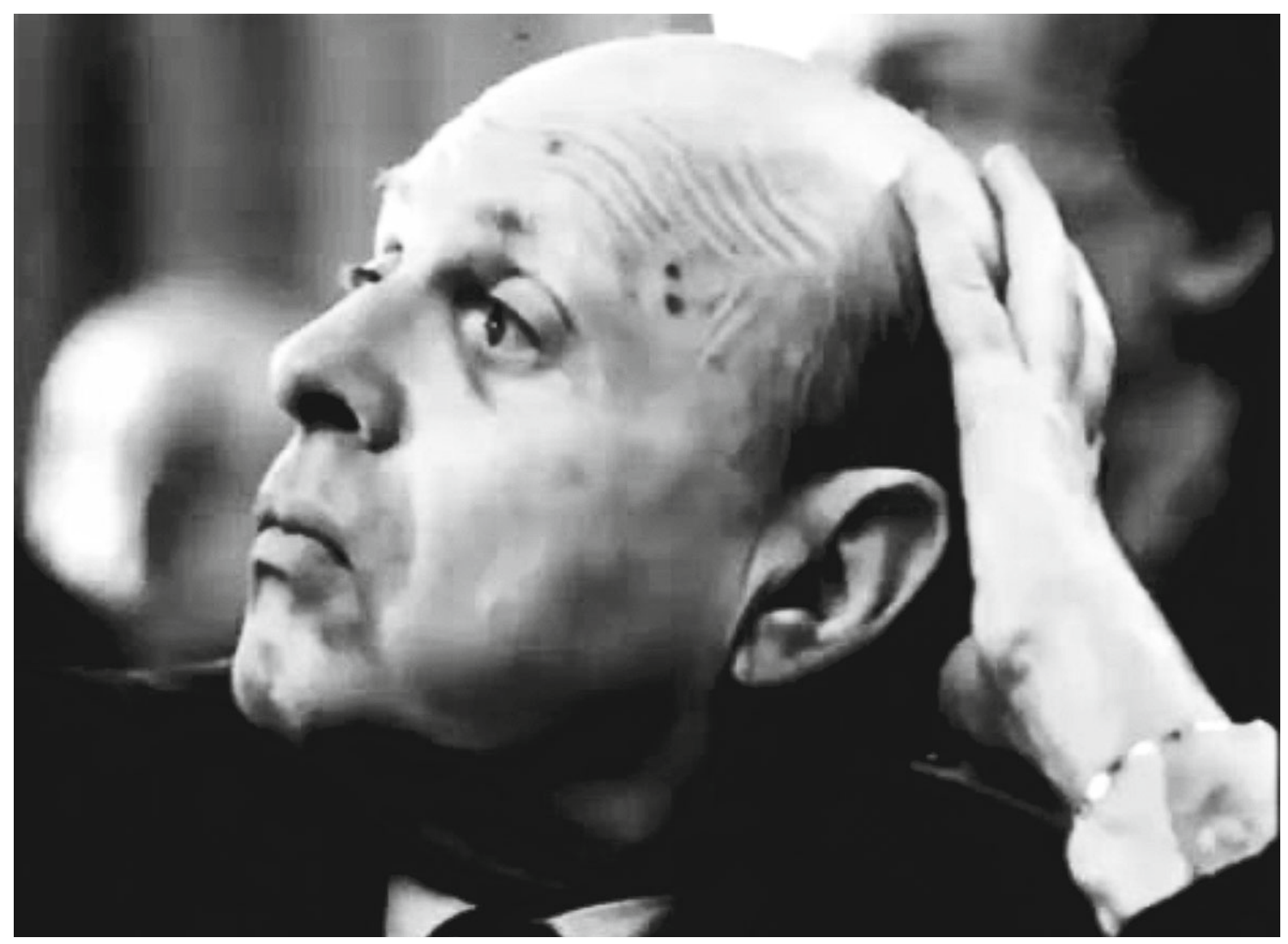

October 19, 2019 is the 100th birthday of Issak Markovich Khalatnikov, a prominent theoretical physicist, whose career started under close supervision of Lev Landau. Khalatnikov is an author of more than 300 fundamental research papers (many of them having been published in JETP, which we are duly proud of) and books on low-temperature physics, quantum electrodynamics, theory of gravitation, and cosmology. His work on computational methods made an essential contribution to the success of postwar Soviet defense programs. Most of Khalatnikov's papers belong to the hall of fame of scientific achievements on the XX and XXI centuries. An impressive list of Khalatnikov's publications, available at http://www.itp.ac.ru/ru/persons/khalatnikov-isaakmarkovich/, starts in the 1940s and extends to the present time. One of his recent papers is published in this issue of JETP. The subjects of other contributions to this anniversary issue reflect the width of Khalatnikov's research interests. Many of these contributions are presented by stuff members of the Landau Institute for Theoretical Physics, an institution that was created and for a long time headed by Khalatnikov. His brilliance in evaluating the academic potential of every scientist allowed him to gather a constellation of remarkable theoretical physicists and mathematicians in Chernogolovka, who made fundamental contributions to the development of science on a worldwide scale. It was due to his dedicated efforts that cooperation with foreign colleagues was successful since the moment of establishing the Landau Institute.

We thank all contributors to this issue and join them in wishing Isaak Markovich Khalatnikov good health and many more scientific achievements. 